\title{
Notes from the EC'14 Program Chairs
}

\author{
VINCENT CONITZER \\ Duke University \\ and \\ DAVID EASLEY \\ Cornell University
}

This short document describes the process used to create the EC'14 program, as well some comments on our experience. This year the conference, which had been called the ACM Conference on Electronic Commerce, was renamed the ACM Conference on Economics and Computation to better reflect the actual makeup of the papers to be presented at the conference. The acronym EC was retained and EC'14 is the fifteenth iteration of the conference.

Since 1999 the ACM Special Interest Group on Electronic Commerce (SIGecom) has sponsored the leading scientific conference on advances in theory, systems, and applications at the interface of economics and computation, including applications to electronic commerce. EC'14 kept the three-track system that was used in previous years. These three tracks are:

- TF: Theory and Foundations

-AI: Artificial Intelligence and Applied Game Theory

-EA: Experimental, Empirical, and Applications

On the Senior Program Committee, we had 15 members for the TF track, 8 members for the AI track, and 8 members for the EA track. This makeup of the SPC in terms of track assignments was the same as last year. Of course, the actual membership of the SPC varied from last year and we made an effort to keep the number of SPC members who had served on the committee for several years to a minimum. SPC members were asked to make suggestions for the PC and we ended up with 130 PC members.

Authors were invited to submit papers and to designate one or two of the tracks for their paper. The call for papers attracted 290 distinct submissions that were deemed to satisfy the formatting requirements. Of these submissions, 212, 73, and 63 chose the labels TF, AI and EA respectively, with 58 papers choosing dual tracks. One issue to note is that several papers, particularly from authors unfamiliar with the process of submitting to computer science conferences, initially contained errors in formatting or in length. Where possible, we helped authors fix these errors, but some thought should be given to how to reduce the initial errors and to the potential inequities of allowing authors to revise the formatting (but generally not the content) of their paper after the submission deadline.

We then asked SPC and PC members to indicate papers that they wanted to review, papers that they were willing to review, papers that they did not want

Authors' addresses: conitzer@cs.duke.edu, dae3@cornell.edu 
to review, and papers with which they had a conflict. We then obtained an initial assignment of (S)PC members to submitted papers using a linear program, based on a compatibility score between each (S)PC member and paper. This compatibility score was based on bids and keywords. We then further refined this assignment by hand.

Each PC member was asked to review a maximum of 8 papers. The number of papers assigned to SPC members varied significantly, but none of them were asked to handle more than 24 papers. Each submitted paper was reviewed by at least three PC members and at least two SPC members on the basis of scientific novelty, technical quality, and importance to the field.

We followed essentially the same review process that was used last year for EC'13. PC members were given just over three weeks to review their assigned papers. Their reviews consisted of written comments and numerical ratings. This was followed by initial discussion that focused on getting the most useful reviews to the authors for the feedback phase. Then, there was a period of two days for authors to provide feedback on the reviews, with this feedback limited to 500 words. Then, the PC members, SPC members, and program chairs engaged in ten days of earnest discussion of the papers, reviews, and feedback. Most papers had extensive discussions, although papers for which the decision seemed obvious after the initial reviews and feedback tended to have less discussion. Overall, both the quality of the reviews and the level of the discussion were very high.

At the end of this process, SPC members made recommendations about which papers to accept. They also wrote meta-reviews for most papers, summarizing the discussion that took place. Finally, the program chairs made final decisions, taking into account SPC members' recommendations, the discussion on each paper, the reviews and numerical scores for papers, and the overall balance of the final program.

At the end of this process 80 papers were selected for presentation at the conference. This is an expansion from the 72 papers accepted last year. However, due to the large increase in the number of submissions, the acceptance rate fell from about $30 \%$ to about $28 \%$. We feel that each of the 80 accepted papers is a strong paper and, in fact, several of the papers that we could not accept were also very good papers. If the number of high quality submissions continues to rise, the community will need to consider whether to allow even more high quality papers to be rejected, or to revise the structure of the conference.

Papers will be presented in a mix of plenary and parallel sessions, with more parallel sessions than in past years because of the greater number of papers on the program. To emphasize commonalities among the problems studied at EC, and to facilitate interchange at the conference, sessions were organized by topic rather than by focus area, and no indication of a paper's focus area(s) appears in the proceedings or will be given at the conference. We obtained an initial clustering of papers into sessions by solving an integer program, based on a compatibility score between each pair of papers. This compatibility score was based on (S)PC bids that the papers had in common, as well as keywords. We then further refined the sessions by hand. (This process was previously used by one of the program chairs for scheduling the AAMAS'12 program; in both cases, Dave Shield provided 
valuable assistance.) Papers with high quality and broad appeal were selected for the plenary session talks. Some of the conference's technically strongest work addresses smaller cross-sections of the community, and so will appear in parallel sessions.

Of the 80 selected papers, 60, 19, and 17 have labels TF, AI, and EA, respectively. 56 of the accepted papers will be published in the conference proceedings. For the remaining 24, at the authors' request, only abstracts are included along with pointers to full working papers that the authors guarantee to be reliable for at least two years. This option accommodates the practices of fields outside of computer science in which conference publishing can preclude journal publishing. We expect that many of the papers will appear in a more polished and complete form in scientific journals in the future.

In addition to the main technical program, EC'14 will feature three workshops and four tutorials, joint sessions with the co-located NBER and Decentralization conferences, a poster session, a talk by ACM SIGecom Doctoral Dissertation Award winner Balasubramanian Sivan, and keynote talks by:

- Matthew Jackson: Centrality, Gossip, and Diffusion of Information in Networks - Kevin Leyton-Brown: Pragmatic Algorithmic Game Theory

Finally, following the guidelines for the ACM EC paper awards, we nominated six papers for the best paper award, with two of these also being eligible for the best student paper award. We selected a best paper award committee consisting of Felix Brandt (chair), Itai Ashlagi, and Mohammad Mahdian. The best paper and best student paper awards will be announced at the conference.

We want to thank EC'14 general chair Moshe Babaioff and the rest of the organizing and program committees; SIGecom (especially David Parkes); the organizing committees of the co-located NBER and Decentralization conferences; Preston McAfee and Eva Tardos, program co-chairs for EC'13, for the notes [McAfee and Tardos 2013] and advice that they provided to us; Dave Shield for much help with running Confmaster; and Ann Marie King for help with the website. We look forward to seeing most of the community in Palo Alto for a very exciting program!

\section{REFERENCES}

McAfee, R. P. And Tardos, É. 2013. Notes from the EC'13 program chairs. SIGecom Exchanges 12, 1, 2-7. 